\title{
Imaging Analysis of Prosthesis Angle after Hip Replacement with Direct Anterior Approach in Lateral Position
}

\author{
Daojian Zhang (D, Liping Pan, Talatibaike Maimaitijuma, Heng Liu, and Hao Wu \\ Peking University First Hospital, Beijing 100034, China \\ Correspondence should be addressed to Daojian Zhang; 04435@pkufh.com
}

Received 6 January 2021; Revised 29 January 2021; Accepted 5 February 2021; Published 17 February 2021

Academic Editor: Zhihan Lv

Copyright ( 92021 Daojian Zhang et al. This is an open access article distributed under the Creative Commons Attribution License, which permits unrestricted use, distribution, and reproduction in any medium, provided the original work is properly cited.

\begin{abstract}
The use of lateral DAA-THA for the treatment of end-stage hip disorders has good recent clinical efficacy, does not require special surgical beds and traction equipment, uses traditional surgical instruments, reduces the requirements for surgical beds and surgical instruments, enters through the nerve and muscle anatomical gap without cutting any muscle or nerve tissue, is minimally invasive, and has good surgical maneuverability, low bleeding, low postoperative pain, short hospitalization time, and rapid recovery. It is a safe and effective minimally invasive procedure because of its light weight, short hospital stay, and rapid recovery. In this paper, we used imaging to observe the angle of the posterior prosthesis. And the results showed that hip arthroplasty using the direct anterior approach improved hip mobility in early stages compared with other approaches and reduced pain. The direct anterior approach and length between total hip arthroplasty using direct lateral and posterior lateral approach and partial data (surgical time, blood loss, etc.) were significantly worse than those using direct forward approach. In addition, the direct anterior approach to total hip arthroplasty is subject to a learning curve and requires at least 33 cases of experience to achieve a lower complication rate.
\end{abstract}

\section{Introduction}

Total hip arthroplasty (THA) is an effective treatment for end-stage hip disorders to correct deformity, relieve pain, and reestablish hip function. In the past, the posterolateral approach (PLA) or the direct anterior approach (DAA) was often used for THA [1]. The DAA is based on the standard nerve and muscle gap approach, which can complete hip replacement surgery with less surgical trauma and is in line with the concept of minimally invasive surgical techniques. The DAA is based on the standard nerve and muscle gap approach and can be used to perform hip replacement surgery with less trauma [2]. The Hueter interval (sutures/ rectus femoris-vastus lateralis) is a true neurovascular intermuscular interface and has become a minimally invasive approach for hip replacement [3]. The aim of minimally invasive surgery is to achieve the same or better clinical outcome than conventional surgery with less trauma [4]. Under the guidance of minimally invasive concept, the entire surgical procedure is not only about small surgical incision, but also about minimizing the damage to the neuromuscular and other tissues, so that patients can obtain clinical results such as less trauma, less bleeding, and faster recovery [5-7]. This reduces the risk of postoperative dislocation and reduces postoperative-related activity restrictions, enabling patients to leave bed for weight-bearing activities pain-free on the second postoperative day or even on the day of surgery, which is conducive to rapid postoperative recovery.

The indications include aseptic necrosis of the femoral head (Ficat stage III-IV), osteoarthritis of the hip joint secondary to formal conservative treatment, and initial unilateral total hip replacement [8]. Contraindications include severe cardiopulmonary insufficiency that cannot tolerate surgery or anesthesia, previous history of hip surgery or artificial hip revision surgery, severe proximal femoral deformity, severe osteoporosis, osteoarthritis secondary to hip dysplasia (Crowe type III-IV), hip infection or hidden or overt infections in other parts of the body, and body mass index $(\mathrm{BMI})>30 \mathrm{~kg} / \mathrm{m}^{2}$ [9]. Obese patients are 
avoided in the early learning curve for beginners [10], and the range of indications can be expanded with experience and proficiency in surgical operation, as well as improvements in surgical access and surgical instruments, but DAA should be selected with caution in patients with considerable muscle development (e.g., weight lifters) [11].

Before the DAA surgery, the position of the greater trochanter and the anterior superior iliac spine was located, and the incision was made from the anterior superior iliac spine $2-3 \mathrm{~cm}$ downward and outward, with a length of $8-10 \mathrm{~cm}$ in the direction of the fibular tuberosity, and the skin and subcutaneous tissues were incised layer by layer, and the broad fascia and muscle membranes were incised to expose the muscle gap between the broad fascia tensor and the suturing muscle. The Hueter gap was fully exposed by placing two pulling hooks on the lateral side and one on the medial side of the muscle gap, as shown in Figure 1 [12]. The ascending branch of the spinolateral femoral artery is identified and separated, and ligation or electrocoagulation is performed, which means that the anterior joint capsule can be reached. The anterior joint capsule is incised in an "I" shape to expose the hip joint. The femoral head is removed using a double-pass method, which facilitates the removal of the femoral head. At the junction of the base of the femoral neck and the greater trochanter, the femoral neck is cut with a swing saw perpendicular to the femoral neck, followed by a secondary osteotomy at a distance of approximately $1 \mathrm{~cm}$ to remove the femoral head and osteotomy block [13]. Two Hohmann hooks were placed at the acetabulum to retract the joint capsule, iliopsoas tendon, and soft tissues to fully expose the acetabulum. The acetabulum was cleaned of soft tissues, the glenoid labrum and the periacetabular rim were removed, and the acetabulum was filed with a special eccentric acetabular file until the subchondral bone was uniformly bleeding. To facilitate exposure of the proximal femur, the bed was lowered caudally by $30-40^{\circ}$, and the proximal femur was retracted from the posterior to the anterior aspect of the acetabulum with a bone hook on the operated limb, and the posterior lateral joint capsule, the joint tendon of the internal closed foramen, the pear-shaped muscle, and the external closed foramen muscle were gradually released by the surgical assistant with internal retraction, gentle posterior extension, and external rotation of the hip joint to maintain soft-tissue tension until satisfactory exposure of the proximal femur was achieved [14-17]. During the operation, a scraper with a curved spoon or an eccentric handle femoral drill was used to open the medullary cavity, and an eccentric medullary file was used to expand the medullary cavity one by one to the appropriate size, rotate to see a good inlay without micromovement, install a trial mold and compare the length of both lower limbs, maintain an appropriate anterior tilt angle to gradually insert a suitable femoral prosthesis, install a femoral head prosthesis, reposition the hip joint to see a good elasticity and stability, and place a negative pressure drain and close the wound after pulse irrigation of the incision [18-21].

The DAA does not damage the muscles around the hip joint, does not cause significant damage to the posterior structures of the hip capsule, and has a better postoperative balance of lower limb muscle strength than the traditional approach, so it has the accuracy of prosthesis placement and good postoperative hip stability, reducing the risk of postoperative soft tissue imbalance caused by periprosthetic muscle injury and postoperative hip dislocation caused by improper placement of the prosthesis [22]. Daily activities such as squatting, sitting on a low stool, and bending to put on socks and laces can be performed in the early postoperative period, and no special protection is needed for the postoperative hip joint [23-25]. In this paper, we used imaging to observe the angle of the posterior prosthesis and showed that hip arthroplasty using the direct anterior approach improved hip mobility in early stages compared with other approaches and reduced pain. The direct anterior approach and length between total hip arthroplasty using direct lateral and posterior lateral approach and partial data (surgical time, blood loss, etc.) were significantly worse than those using direct forward approach. In addition, the direct anterior approach to total hip arthroplasty is subject to a learning curve and requires at least 33 cases of experience to achieve a lower complication rate.

\section{Lateral Microanterior Approach Hip Arthroplasty}

In this work, the angle of the posterior prosthesis by total hip arthroplasty using the direct anterior approach resulted in better hip mobility and less pain in the early postoperative period compared with other approaches.

\subsection{Traditional THA Surgical Approach. Artificial hip re-} placement has a history of nearly 100 years and many studies on its surgical approaches have been reported in the literature, leaving us with a valuable asset. The posterior lateral approach has a high probability of posterior dislocation, the anterior lateral approach has a high rate of anterior dislocation, and the lateral approach has a high rate of abductor weakness. The following principles should be followed in selecting the approach for hip replacement surgery: (1) it is easy to reveal the acetabulum and proximal femur, and the incision can be extended up and down; (2) it does not affect the nerve tissue, muscle tissue, and tendons; (3) it is conducive to the placement of the prosthesis; (4) it is conducive to the comparison and adjustment of the length of both lower limbs during surgery; (5) it is conducive to functional recovery; and (6) there are few intraoperative or postoperative complications. Therefore, the choice of the surgical approach is based on the surgeon's personal experience and preference. In terms of medical safety, it is beneficial for the patient to choose the surgical approach in which the surgeon is most skilled. In addition, it is important to evaluate the patient's specific situation, such as whether it is a primary replacement or a secondary revision, the location and extent of the acetabular bone defect, the use of structural or compression bone grafting, previous surgical approaches, and the type of prosthesis. Regardless of the procedure chosen, it is important to achieve adequate exposure of the 


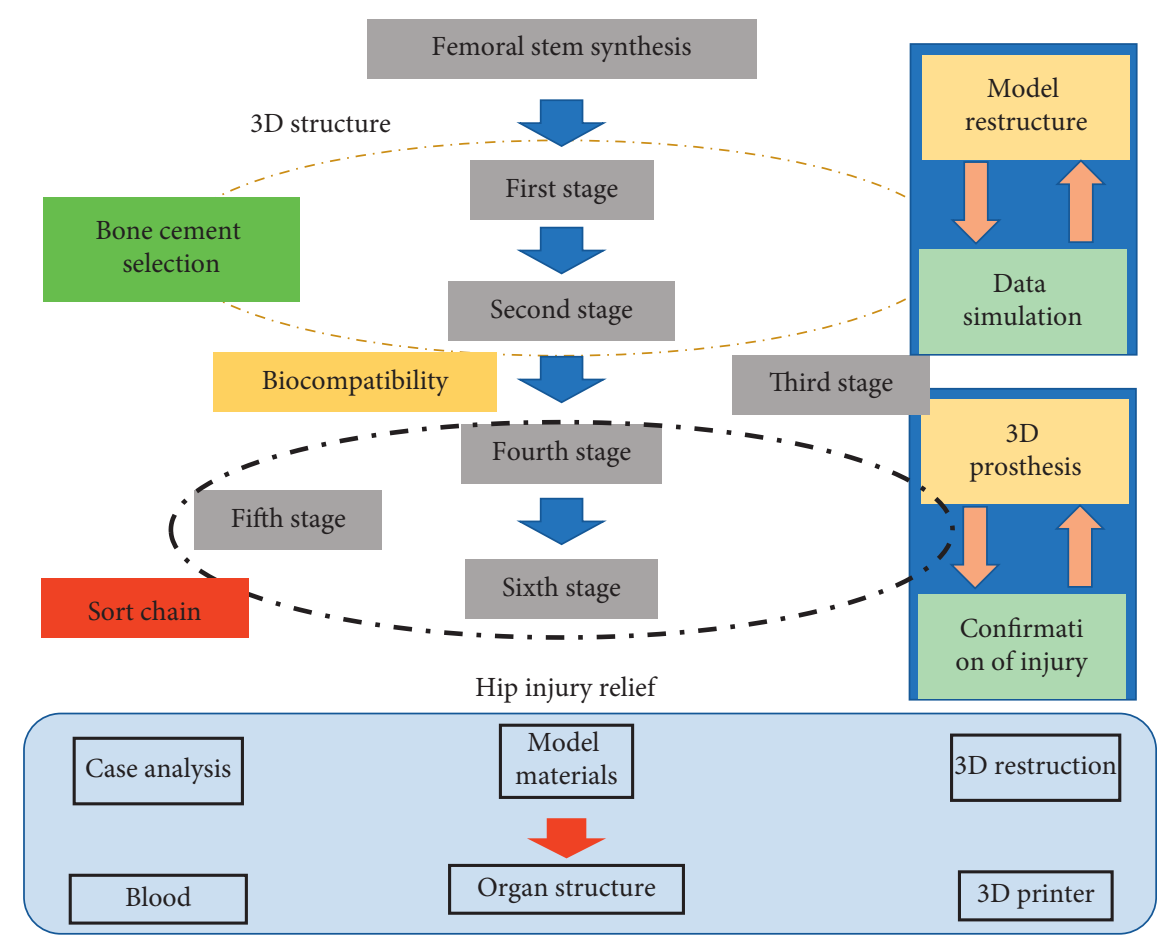

FIGURE 1: The operating procedure of DAA operation.

operative field and to be able to extend the incision whenever necessary to facilitate completion of the procedure.

The procedure is performed as shown in Figure 2, under general or combined hard and lumbar anesthesia, with the patient lying on the general operating table on the healthy side, keeping the transverse axis of the pelvis perpendicular to the general operating bed and ensuring that it is secured so that the anterior aspect is tightly pressed against the pubic symphysis and the posterior aspect is squeezed against the sacrum. The proximal end of the incision starts about $4 \mathrm{~cm}$ below the posterior superior iliac crest and runs parallel to the posterior border of the greater trochanter in the direction of the gluteus maximus muscle fibers, and the distal end of the incision can follow the axis of the femoral stem to the greater trochanter and be about $10-15 \mathrm{~cm}$ long. The posterior edge of the gluteus medius muscle and part of the external rotator muscle group were exposed by blunt finger dissection or electric knife dissection of the broad fascial tensor and gluteus maximus fibers, and the fat on the surface of the muscle group was removed, and the blood vessels were located for electrocoagulation or ligation to stop bleeding, and several external rotators were visible. The terminal branch of the medial femoral artery is noted. The gluteus maximus fascia and the attachment of the broad fascia were dissected, and the short external rotators and the posterior capsule were marked with sutures and reconstructed at the end of the procedure to maintain capsule tone and muscle integrity to reduce the risk of dislocation. The hip joint is continued to be released, and the hip joint is flexed at $90^{\circ}$ and internally rotated so that the sole of the foot is facing upward, and the femoral head can usually be dislocated successfully. If the hip is more difficult to dislocate, a bone hook can be used to lift upward under the femoral neck to reset it.
Do not manipulate the affected limb roughly to prevent spiral fracture of the femoral stem, fully release the joint capsule, and remove all bony redundancies at the posterior edge of the acetabulum to prevent dislocation caused by hip impingement. The sciatic nerve can be palpated on the surface of the innominate muscle and the gluteal muscle during posterior exposure. For the first hip replacement, it is not necessary to dissect the sciatic nerve, which can be protected by bluntly pushing down the gauze or turning back the short external rotation muscle group by cutting it. In a modified minimally invasive approach to the traditional posterior lateral approach, the vascular network behind the greater trochanter of the femur and the arterial branches below the pear-shaped muscle and above the superior distalis muscle are electrocoagulated or ligated before cutting the external rotator muscle group and incising the joint capsule. A small portion of the muscle fibers should be left attached to the femur for electrocoagulation to stop bleeding when the femoral square muscle is cut. In addition, controlled decompression and exposed femoral bone surface can be stopped with bone wax to reduce bleeding. As experience is gained, the posterior lateral approach incision can be shortened and minimally invasive operations can be performed to help accelerate recovery.

2.2. Posterolateral Approach to the Hip Joint. The indications and contraindications for initial THA using DAA are not standardized and depend on the operator's proficiency in the traditional total hip replacement approach and the patient's overall evaluation. The posterolateral approach is one of the traditional surgical approaches for hip replacement, which has the advantages of good familiarity with the orthopaedic 


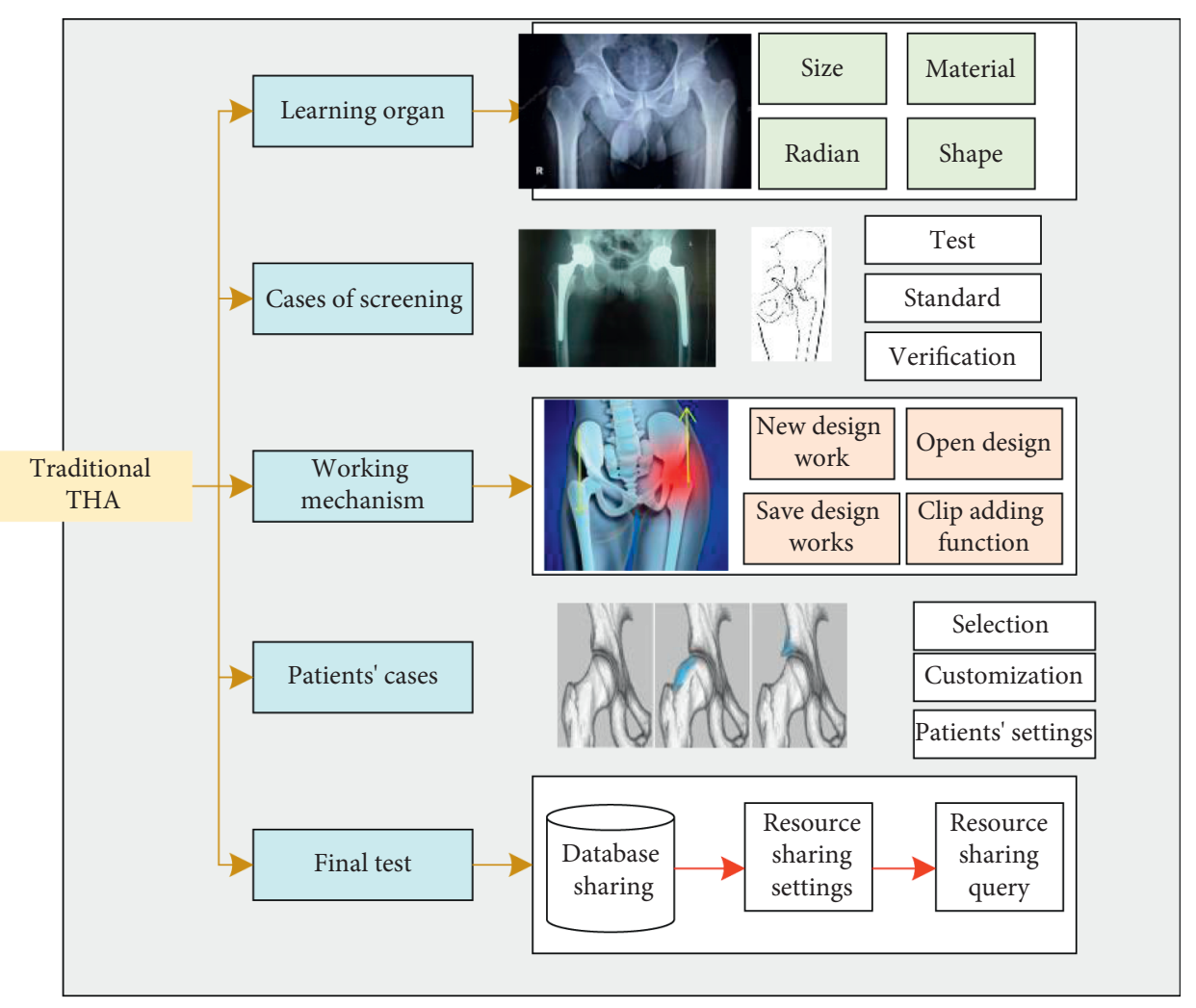

Figure 2: Traditional THA surgical approach.

surgeon and good exposure of the femoral side, while DAA has a long learning curve; therefore, the early indications for beginners are more stringent, including aseptic necrosis of the femoral head (Ficat stage III-IV), osteoarthritis of the hip joint that has been ineffective after regular conservative treatment, and initial unilateral total hip replacement. In previous studies, DAA-THA was classically performed using a special surgical bed and a supine position with lower limb traction. In contrast to conventional surgery, the special surgical bed requires a higher cost and specific complications such as ankle fractures. In our case, the patient was laterally positioned on a regular surgical bed, and intraoperative traction bed manipulation was not required, nor was surgical bed deformation required to achieve surgical requirements, as the proximal femur could be exposed only by inversion, hyperextension, and external rotation of the affected limb by an assistant, which facilitated the development of the DAA technique. The VAS pain scores of our patients showed significant improvement in postoperative pain, and the postoperative hip Harris scores improved considerably at 1 , 3 , and 6 months postoperatively and at the last follow-up.

Theoretically, the direct anterior approach does not damage the periprosthetic muscles and does not require reconstruction of the integrity of the periprosthetic muscles after the prosthesis is installed, and the operative time and intraoperative bleeding are less than those of the posterolateral approach and other traditional hip surgical approaches. However, when comparing the supine DAA and the traditional posterolateral approach (PLA), the average operative time was $84.3 \pm 12.4 \mathrm{~min}$ in the DAA group and
$60.5 \pm 12.4 \mathrm{~min}$ in the PLA group; the average bleeding in the DAA group was $391 \pm 206 \mathrm{~mL}$ and $191 \pm 107 \mathrm{~mL}$ in the PLA group, with statistically significant differences between the two groups $(P<0.0001)$. Subsequently, we compared the operative time and bleeding volume of the same surgeon using two surgical approaches (supine DAA and conventional PLA approach) and reached similar conclusions. We compared the direct anterior approach with the posterior lateral approach and found that the Harris hip function score, UCLA score, and gait parameters were better in the DAA group than in the PLA group after follow-up. The posterolateral approach is one of the traditional surgical approaches for hip replacement, which has the advantages of good familiarity with the orthopaedic surgeon and good exposure of the femoral side, whereas the supine DAA has a long learning curve and therefore the operative time and bleeding increase accordingly. The mean operative time in this study was $58 \pm 8.2 \mathrm{~min}$, which is similar to the PLA operative time in the literature. When switching from conventional PLA to supine DAA, the surgical bed had to be adjusted when the proximal femur was exposed, and the acetabular side was handled differently from the conventional habit; however, when switching from conventional PLA to lateral DAA, the habit of acetabular side handling did not change significantly, and the view exposed in lateral DAA was not significantly different from that of conventional PLA, and the affected limb could be flexibly posteriorly extended, externally rotated and lateral femoral release is more convenient than supine DAA, which is conducive to shortening the learning curve. In addition, the mean blood 
loss in this study was $320 \pm 82.0 \mathrm{~mL}$. However, it was difficult to assess whether there was a difference in bleeding between lateral DAA and PLA and supine DAA because tranexamic acid was left in the joint cavity after surgery and there was no control group in the study subjects.

\subsection{Analysis of Complications of DAA. Deep Venous} Thrombosis (DVT) is one of the common complications after hip and knee arthroplasty, which can cause fatal pulmonary embolism in severe cases and increase the economic and clinical burden of patients. Even though experts and scholars have formulated more detailed guidelines and recommendations for standardized prevention, such as physical and pharmacological prevention, it is still difficult to eradicate its occurrence. Long-term bed rest remains one of the most common high-risk factors for lower extremity deep vein thrombosis. If patients cannot leave bed early after lower extremity arthroplasty, the intravascular blood viscosity will increase and blood flow will be slow; the trauma caused by arthroplasty makes patients reluctant to move the affected limb due to pain after surgery, and the muscle contraction will be weakened, which directly leads to the weakening of the "muscle pump." In addition, elderly patients with reduced cardiopulmonary function and slow venous blood flow in the lower extremities are prone to DVT formation. Pulmonary embolism has a high mortality rate and prevention is the key, so it is important to have a rapid recovery after arthroplasty. They performed chemoprevention of DVT in 963 patients after total hip and knee arthroplasty from March 2010 to July 2013, followed by imaging, and the incidence of DVT was $17.96 \%$. They reported an overall prevalence of DVT of $4.8 \%$ in 459 patients (516 hips) with a lower extremity mechanical compression device. In our group, one patient $(0.3 \%)$ presented with lower extremity swelling 2 days after surgery, which was confirmed as DVT by B-ultrasound and cured by placement of a filter in the vascular department. One case of intermuscular vein thrombosis was cured by anticoagulation. To prevent and reduce the occurrence of DVT, the patient's coagulation status should be assessed before surgery and treated in a timely manner, and oral rivaroxaban should be routinely administered until 5 weeks after surgery. Postoperative infection after total hip replacement includes superficial infection of the superficial port and deep tissue infection. Incisional hematoma and superficial infection of the incision may occur in the early postoperative period and should be strictly stopped at the end of the procedure to avoid the presence of active bleeding. Because the incision is located anterolaterally, there is no significant intrinsic pressure to stop the bleeding, so the hematoma that forms may be greater compared to other hip approaches.

Pulling the hook to pull the incision excessively may damage the skin, subcutaneous tissue, and muscle layer, and the surgeon must always be aware of the need to protect the skin during surgery, especially during lateral femoral manipulation. Obese patients with a Body Mass Index (BMI) $>30 \mathrm{~kg} / \mathrm{m}^{2}$ are an important challenge for the surgeon in DAA-THA, especially in supine DAA-THA in the supine position. Due to the large exposure and accumulation of abdominal adipose tissue, muscle and adipose tissue tend to crowd into the incision, resulting in inadequate exposure of the surgical site, making the operation extremely difficult, and making the skin around the incision appear more fragile, also increasing the risk of postoperative superficial incision infection and proximal femoral fracture due to contusions of the skin. In obese patients, there are folds in the skin that occur during postoperative hip flexion, making it difficult to cover the incision dressing. Also folds at the groin cause moist incisions and harbor more bacterial microorganisms, which also contribute to incisional infections. They concluded that obese patients $\left(B M I>30 \mathrm{~kg} / \mathrm{m}^{2}\right)$ significantly increased operative time, wound complications, and hospital days. In our study, we noted that in lateral DAA-THA, no significant increase in surgical difficulty was seen due to the gravitational effect of the abdominal adipose tissue around the hip joint as well as the subcutaneous adipose tissue, which moved away from the surgical field. In this group of cases, 32 (39 hips) obese patients with BMI $>30 \mathrm{~kg} / \mathrm{m}^{2}$ underwent DAA-THA, and superficial wound infection occurred in all 3 (3 hips) obese patients, and the incidence of incisional complications was $0.9 \%$, manifesting as erythema and oozing around the superficial incision, probably related to fat liquefaction. There was no reoperative management and it healed after several dressing changes, so we did not list obese patients with BMI $>30 \mathrm{~kg} /$ $\mathrm{m}^{2}$ as a contraindication in this group of cases, taking fully into account the advantages of lateral recumbency.

However, for beginners, avoid selecting obese patients for DAA-THA. To prevent infection, take a careful history and physical examination before elective hip replacement surgery to exclude septic skin lesions, prostatitis, pelvic inflammatory disease, urethritis, pneumonia, gingivitis, and periodontitis. If these diseases are combined, wait for the inflammation to heal before performing surgery to avoid deep-tissue infection. We routinely administered secondgeneration cephalosporins intravenously half an hour before the start of surgery and prophylactic anti-infection for 1-2 days after surgery. The drainage tube was removed when the incisional drainage was $<50 \mathrm{~mL}$ within $20-48$ hours after surgery, and the patient was removed from bed for weightbearing activities on the second postoperative day or even on the day of surgery under the guidance of the rehabilitation physician. If there is purulent drainage in the drainage tube or redness and fever in the incision after surgery, promptly perform bacterial culture, blood culture, drug sensitivity test, joint puncture fluid culture, etc., and deal with the situation accordingly. If deep infection or hip joint infection occurs after total hip replacement, it will bring huge economic and psychological burden to the patient, implying the possibility of reoperation and causing death of the patient in serious cases. There are many reasons for the occurrence of infection, including patient factors, medical factors, nursing factors, and improper use of antimicrobial agents. Medical factors are one of the most important causes of postoperative hip infection, such as poor aseptic concept, lack of strict aseptic operation during surgery, rough surgical procedure, large surgical trauma, long-term exposure of incision, and 
excessive bleeding. The acute infection is relatively superficial and can be controlled and cured by the use of sensitive antimicrobial agents or wound debridement and drug replacement according to the results of drug sensitivity; the chronic infection is deeper and the use of antimicrobial agents and extended debridement often cannot change the regression, so it is necessary to perform antibiotic cement spacing and wait for the normalization of the inflammatory infection indexes. After that, hip revision should be performed. Deep infection after total hip arthroplasty is a catastrophic complication for patients, but with experience the infection rate decreased significantly, and no deep incision infection occurred in this group of cases. The lateral DAA-THA learning curve increases the operative time and bleeding volume early on and may also increase the risk of postoperative infection, which decreases significantly as the surgeon gains experience.

\section{Postoperative Prosthesis Imaging Analysis}

3.1. Bone Cemented Acetabular Prosthesis. The success of total hip arthroplasty was initially attributed to the ability of the polymethylmethacrylate cement to securely fix the prosthesis to the bone, as shown in Figure 3, although the cement manufacturing process continues to improve and the use of biologic prostheses is still the current trend despite the lower overall cost of using cemented prostheses. The main purpose of cemented patellofemoral prostheses is to create a solid mechanical locking effect between the cement and the acetabular prosthesis, ultimately creating osseointegration between the cement and the bone, a mechanism that ensures long-lasting stability of the prosthesis. Bone cemented hip prostheses can be used for total hip arthroplasty in patients of any age; polyethylene prostheses, which are prone to wear, should not be used in young patients and in patients with high postoperative mobility requirements and should be replaced with highly cross-linked polyethylene to reduce polyethylene wear; subchondral bone bleeding in the acetabulum needs to be treated before the application of bone cement, using massive pulse irrigation, suction with a suction device, and pressure in combination with bone. In cases of acetabular bone defects or bone cysts, autologous bone should be taken first and then bone cement should be used to fix the prosthesis after compression bone grafting in the acetabular white, and if necessary, allograft bone or metal patches should be used to obtain a good acetabular socket for cemented prosthesis. In chronic septic arthritis, bone cement with sensitive antibiotics can be used to reduce the risk of recurrent infection. There are generally no contraindications to cemented acetabular prostheses for total hip replacement.

3.2. Biological Acetabular Prosthesis. The high failure rate of using cemented acetabular prosthesis fixation in total hip arthroplasty, as suggested by some studies, has promoted the use of biologic acetabular prosthesis as one of the main methods of hip reconstruction in total hip arthroplasty, as shown in Figure 4. Modern acetabular prosthesis designs are

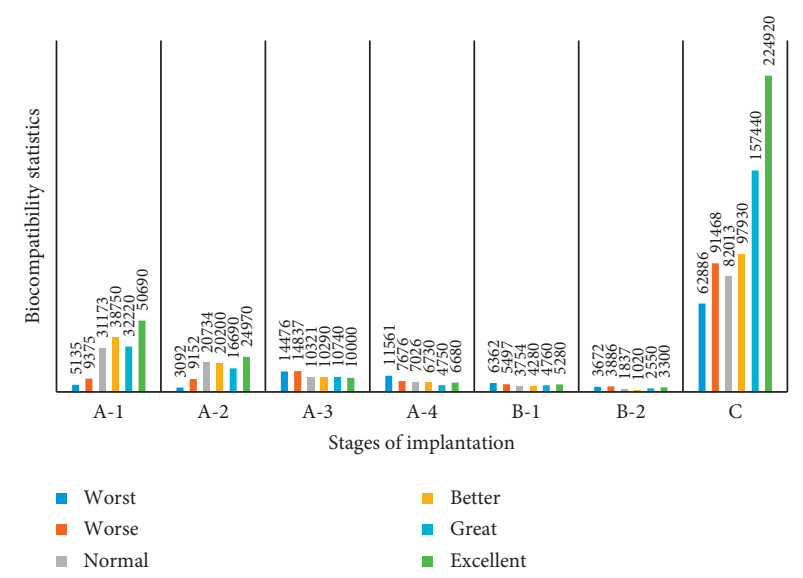

FIgURE 3: Changes in total hip arthroplasty prosthesis.

able to provide adequate initial stability through press-fit and screw fixation of the prosthesis, and the prosthesismarrow-white interface is conducive to intraprosthetic growth and fixation of the bone phase and also exhibits good acetabular prosthesis survival rates. The use of high crosslinked polyethylene in total hip arthroplasty has been effective in reducing the wear rate of biologic acetabular prostheses, even in young patients and those with high postoperative mobility requirements. Polyethylene also improves the survival rate of the acetabular prosthesis. Biologic acetabular prostheses can be used for all primary total hip arthroplasties and revisions, including acetabular dysplasia or osteoarthritis secondary to acetabular impingement, acetabular impingement, femoral head necrosis, and femoral neck fractures. Overall, the initial stability of the biologic acetabular prosthesis and the biologic stability resulting from long-term bone growth into the biologic acetabular prosthesis have improved the survival rate of total hip replacements, and the improved wear resistance of the surface of the acetabular prosthesis and the leap in polyethylene quality have contributed significantly to the better survival rate of the acetabular prosthesis.

3.3. Bone Cemented Femoral Stem. Since the application of bone cement in total hip arthroplasty in the 1960s, the design of some femoral stems has been very successful, but of course there are many more femoral stems that are not as well designed. The long-term stability of cemented femoral stems in hip replacement requires two principles: first, the use of a biomechanical cemented stem as much as possible; second, the surgeon in charge should master the skill of using the cemented femoral stem. The term "cement" in orthopedics refers specifically to polymethyl methacrylate, which is an elastic material and is therefore often compared to cement in construction, but the long-term fixation performance of polymethyl methacrylate is closely related to the bone cement shank design characteristics. More importantly, when the bone cement is stimulated by continuous stress, it will slowly soften, which is a slow process that some cemented femoral stems are designed to take into account, while other cemented femoral prostheses do not take into account this slow process, as shown in Figure 5. 


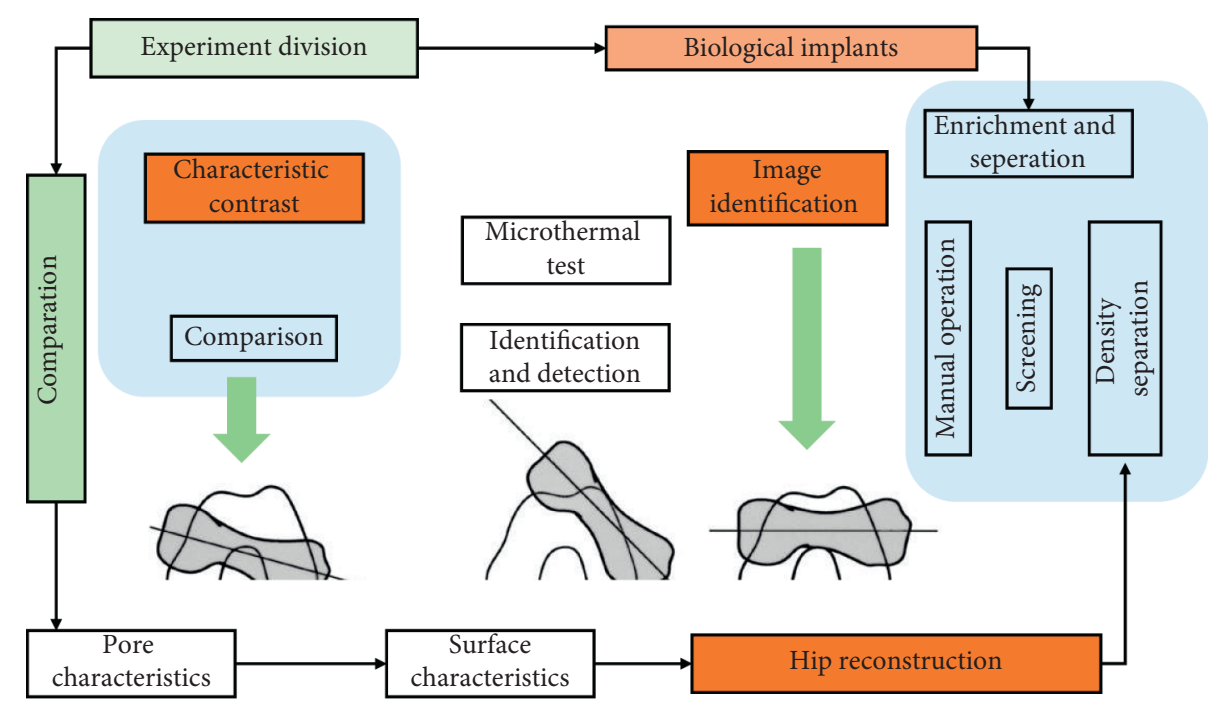

FIgURE 4: Biomimetic acetabular prosthesis for hip reconstruction.

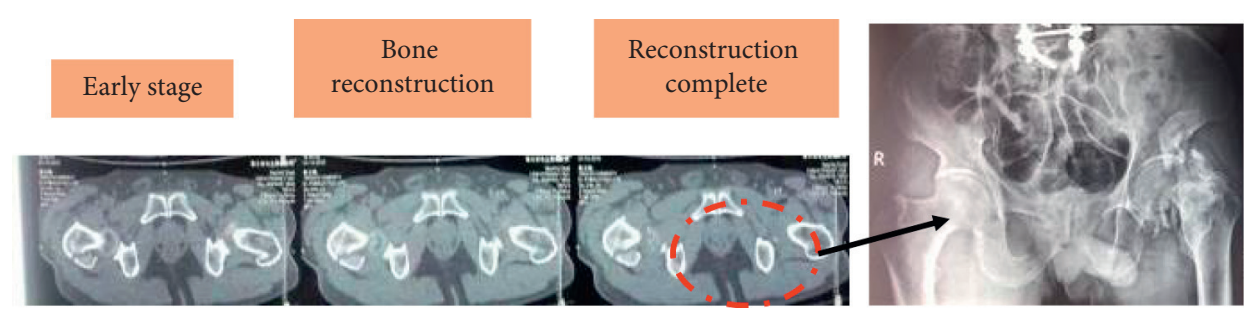

FIGURE 5: Imaging changes of the femoral stalk on CT scan in total hip arthroplasty.

In general, cemented stems are divided into morphologically matched cemented femoral stems and mechanically matched tapered cemented femoral stems. The surface of the tapered cemented femoral stem is polished to reduce the effect of methacrylic acid polymers. When the tapered cemented femoral stem and the cement are affixed, the tapered cemented femoral stem is shaped into a cone with the cement under continuous compression. The fixation characteristics of this cemented femoral stem are such that sinking of the cemented femoral stem is prevented to the greatest extent possible, or at most only slight sinking occurs. Studies using CT 3D reconstructions have found that the cemented femoral stem begins to sink within the first year and slows down over time, with the cemented femoral stem sinking $0.7 \mathrm{~mm}$ within 2 years and only $1.3 \mathrm{~mm}$ by year 10 , which is the expected rate of sinking for a cemented femoral stem that requires long-term stability. Many cemented femoral stems are designed in conjunction with a distal femoral medullary plug so that the cement is not overstressed distal to the femoral medullary cavity. The sinking of the cemented femoral stem in the bone cement is also beneficial, a feature that makes the cemented femoral stem more resistant to torsional forces and thus provides more rotational stability. Comparing the tapered cemented femoral stem to the hybrid cemented femoral stem, the hybrid cemented femoral stem also sinks at long-term follow-up, but the hybrid cemented femoral stem sinks less than the tapered cemented femoral stem. The design of the tapered cemented femoral shank differs from the hybrid cemented femoral shank in that the rough surface of the hybrid cemented femoral shank facilitates the adhesion of the methacrylic polymer to the surface of the cemented femoral shank, bonding the cement to the cemented femoral shank as one piece. Such a cemented femoral stem is more appropriately described as a bonded stem, and micromovement of the cement to the cemented femoral stem should be avoided as much as possible, since even small micromovements can cause damage to the cemented femoral stem due to wear and tear of the cement, eventually leading to loss of stability and aseptic loosening of the cemented femoral stem.

The world's first cemented tapered femoral stem was introduced in 1969 and was first fitted to a living hip in 1970. Data from several joint centers have shown that cemented tapered femoral stems have a clinical prognosis that is no different from other femoral stems in terms of aseptic loosening and revision rates. In addition, because they are cemented and available in different types and eccentric distances, these cemented tapered femoral stems are easier to restore the center of rotation of the hip in primary total hip replacement, which means that the orthopaedic surgeon selects the appropriate eccentric distance and size of the femoral medullary cavity based on the patient's condition and finally decides intraoperatively how deep to install the femoral stem into the femoral medullary cavity based on the length of the lower extremity to be restored. The depth of the 
femoral stem into the medullary cavity is determined intraoperatively according to the length of the lower limb to be recovered. Patients with a larger eccentric distance and a narrower medullary cavity are usually easier to use than patients with a smaller eccentric distance and a larger medullary cavity. The polished, tapered design makes it easier to manipulate during revision. In hip revision, the bone cement of the femoral neck can be removed first, then the cement around the femur can be cleared and the stem slowly removed, then the acetabular side of the prosthesis can be fully exposed, and finally the stem can be reinstalled, either with the same size or with a smaller size. The survival rate of cemented femoral stems is related to the operator's technique and experience with the cement. The goal is to form a tight bone ingrowth between the cement and the patient's femur, so this is achieved through delicate intraoperative surgical manipulation. The initial use of cement fixation should ensure that there is sufficient fixed bone in the patient's femur, and it is also important to irrigate with pulsed pressure prior to fitting the prosthesis. A bone cement gun is placed into the femoral medullary cavity to instill methyl methacrylate, and the methyl methacrylate is pressurized before the cement sets, and then the cemented femoral stem is installed and further pressurized until the methyl methacrylate is completely set.

Cement fixed femoral stem is suitable for all patients receiving primary hip arthroplasty, and tapered cement fixed femoral stem is better than hybrid cement fixed stem. There is no limit to the age or diagnosis of the patient in selecting an appropriate cement fixed femoral stem even in complex cases with the need for anatomical deformation, femoral shortening, and osteotomy. If osteotomy is necessary, it is necessary to press the cement to the osteotomy so that the cement does not enter the endometrium of the osteotomy gap after fixing the osteotomy plate. Cemented femoral stems may also be used in total hip arthroplasty for femoral neck fractures. Cemented fixation for septic hip osteoarthritis has a better long-term prognosis because the addition of antibiotics to the cement reduces the risk of recurrence of postoperative infection. There are no specific contraindications to the use of cemented femoral stems in patients with total hip replacements.

\section{Results and Discussion}

The selection of patients for total hip arthroplasty by the direct anterior approach is based on the level of skill and experience of the orthopaedic surgeon. This means that the direct anterior approach is generally not chosen for patients who require a large interrotor osteotomy and patients who require a subrotor osteotomy. In addition, cases with difficulty in installing a straight femoral stem in direct anterior approach hip arthroplasty should also be taken into consideration, because the subsequent revision may require an increased exposure of the femoral field and is relatively less suitable for direct anterior approach total hip arthroplasty. In addition, patients with significant skin inflammation, skin folds at the hip joint, or fungal skin infections may also be indirectly infected if they undergo direct anterior approach total hip arthroplasty. Recent studies comparing the direct anterior approach with other approaches have also been reported. Studies comparing total hip replacement with the posterior approach have shown that rapid hip function recovery and better gait can be achieved with direct anterior approach hip replacement. A retrospective diagnostic study by MRI found a better level of soft tissue protection with the direct anterior approach than with other conventional approaches. Another study showed that creatine kinase levels (a marker of muscle damage) were relatively low after direct anterior approach hip arthroplasty compared to the posterior approach. A prospective randomized controlled study of direct anterior approach total hip arthroplasty and posterior lateral approach total hip arthroplasty was done and showed that the direct anterior approach group had better functional recovery and lower VAS scores at 3 months postoperatively, as shown in Figure 6.

In the randomized controlled trial of direct anterior approach total hip arthroplasty and backward low invasion approach total hip arthroplasty, the advantage of the former walking gait was seen. In another study, direct anterior approach total hip arthroplasty had an advantage of early functional recovery and relative safety compared with posterior lateral approach total hip arthroplasty, but these advantages were found to have not expanded beyond postoperative two weeks, as shown in Figure 7. The advantage of early and rapid functional recovery is reflected in another subset of cases in which the total hospital stay was significantly shorter in the direct anterior approach group than in the posterior approach group. In another prospective study, where the same postoperative rehabilitation exercises were performed, there was no statistically significant difference in multiple outcomes between direct anterior approach total hip arthroplasty and posterior approach total hip arthroplasty.

Small differences in postoperative gait parameters can be recorded and analyzed by a gait analyzer in patients on the pathway, and most patients with direct posterior approach total hip arthroplasty have a more rigid gait after surgery, as shown in Figure 8. A prospective randomized controlled trial of direct anterior approach total hip arthroplasty versus anterolateral approach total hip arthroplasty showed superior linear analog scale scores after direct anterior approach total hip arthroplasty, and the advantage of the direct anterior approach total hip arthroplasty group lasted for more than 6 weeks. Although many orthopaedic surgeons have demonstrated that X-ray fluoroscopy during direct anterior approach total hip arthroplasty can significantly improve the accuracy of the placement of the acetabular prosthesis, its clinical value is not clear. Although the risk of posterior instability after posterior approach total hip arthroplasty can be reduced to $1-2 \%$ by reconstructing the posterior soft tissue of the hip and using a larger diameter femoral head, the risk of postoperative instability after direct anterior approach total hip arthroplasty is only $0.1 \%-2 \%$. This result indicates that direct anterior approach total hip arthroplasty is significantly better than posterior approach direct anterior approach total hip arthroplasty. Of course, some orthopaedic surgeons believe that direct anterior approach total hip arthroplasty has a higher risk of fracture 


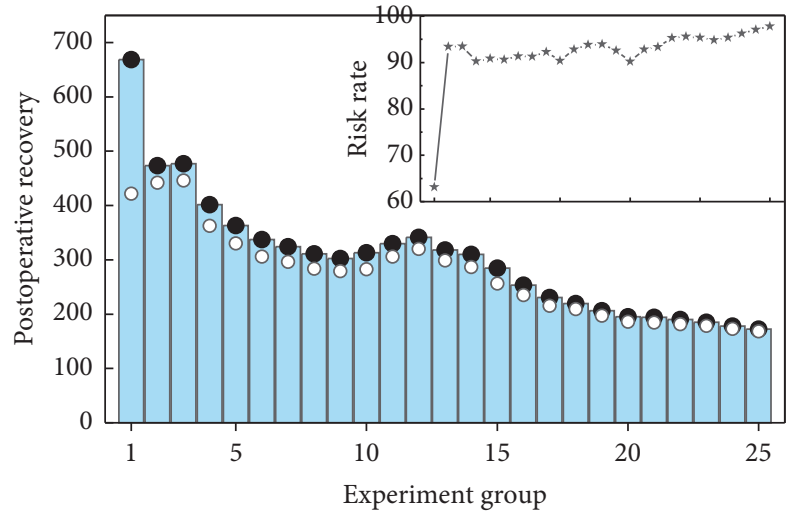

(a)

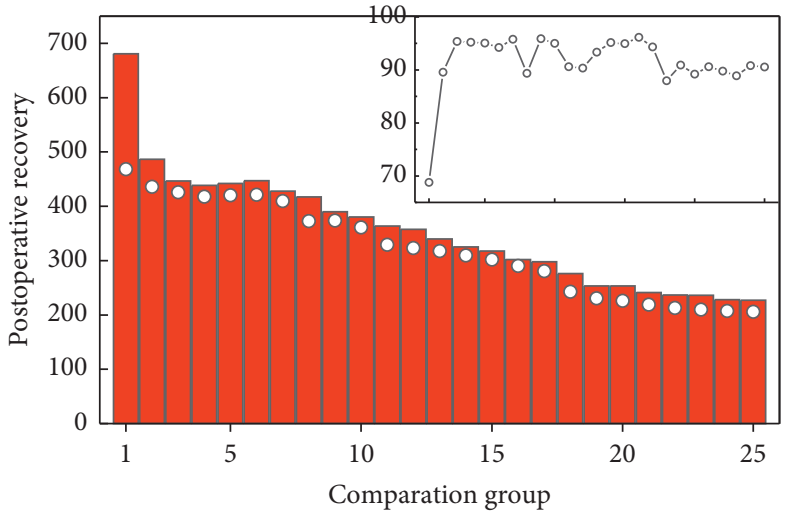

(b)

Figure 6: The degree of functional recovery in the experimental and control groups.

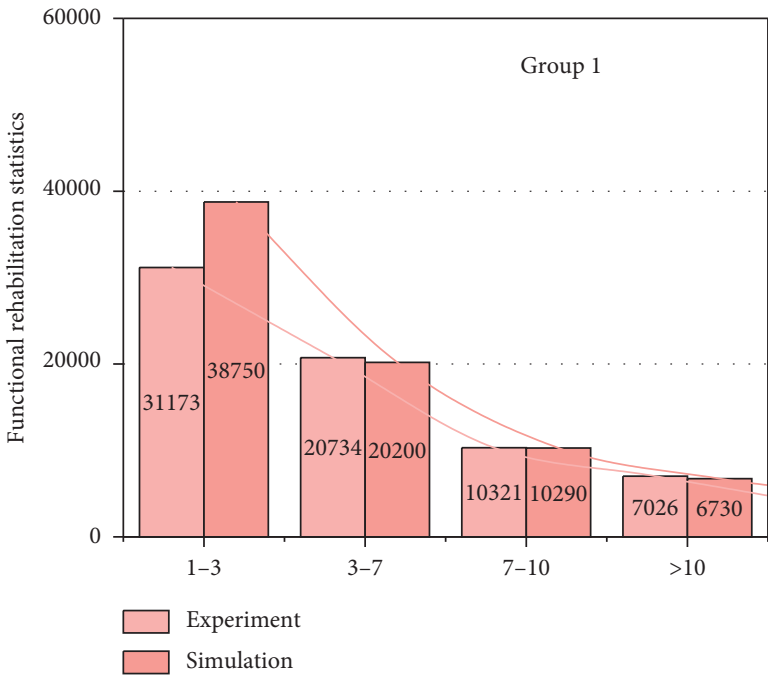

(a)

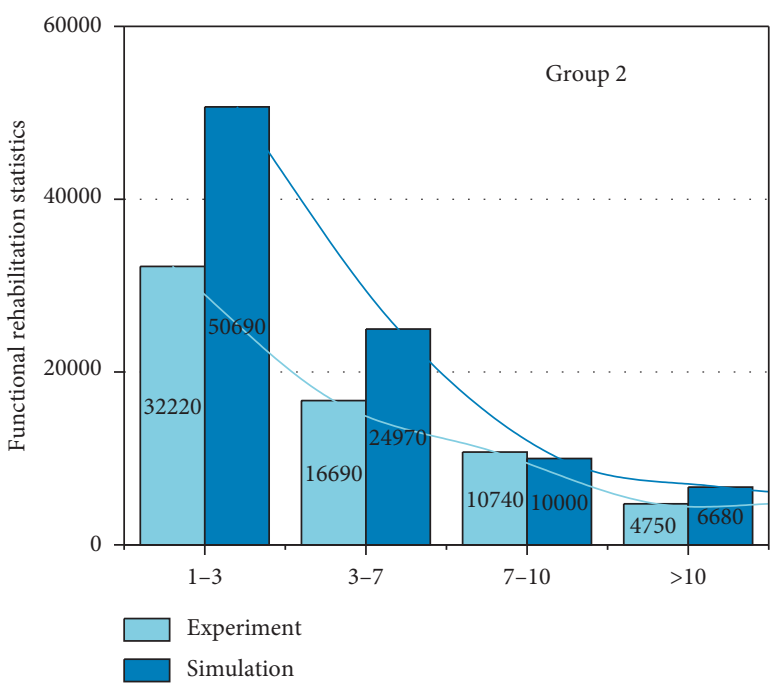

(b)

FIGURE 7: Rapid functional recovery in the early postoperative period.

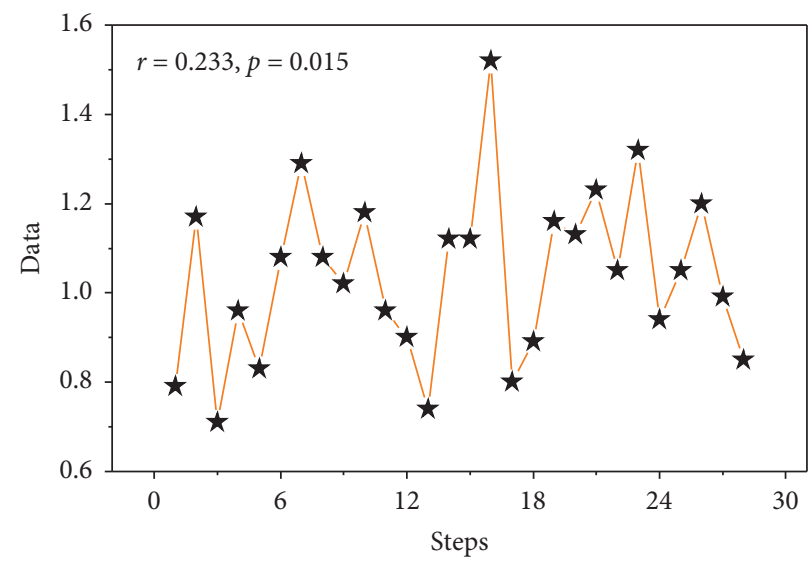

(a)

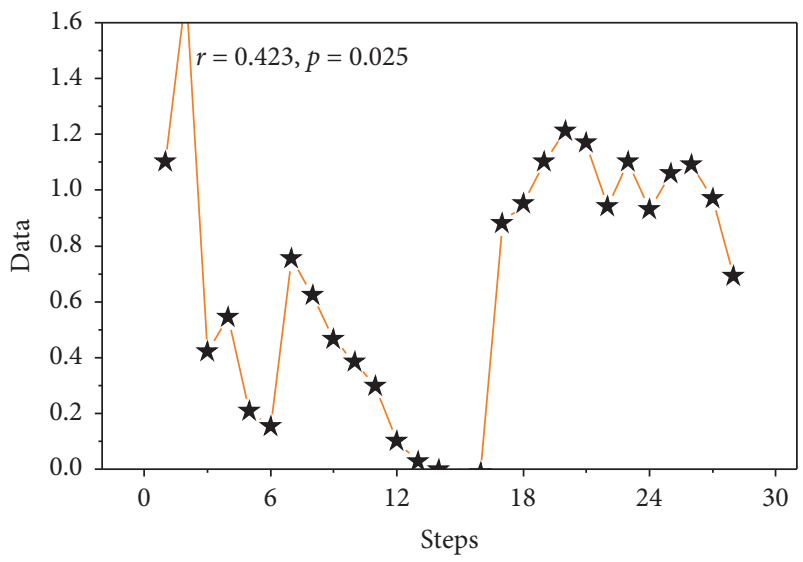

(b)

Figure 8: Continued. 


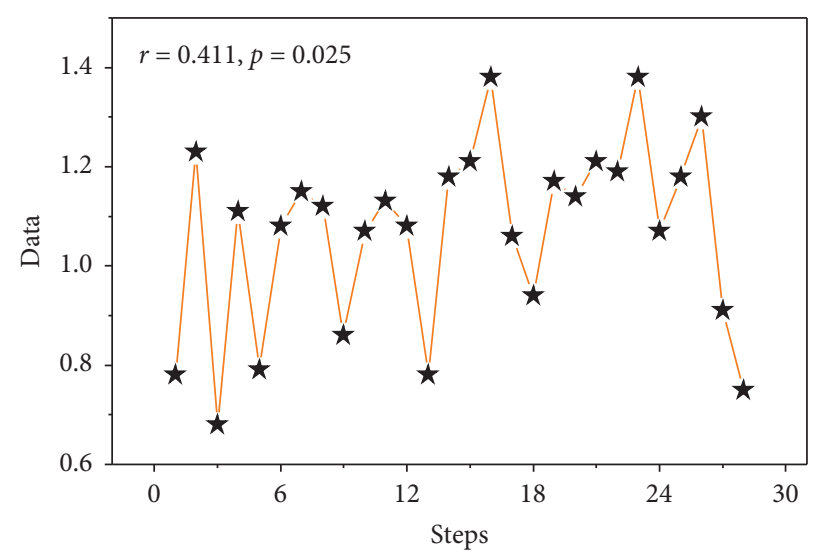

(c)

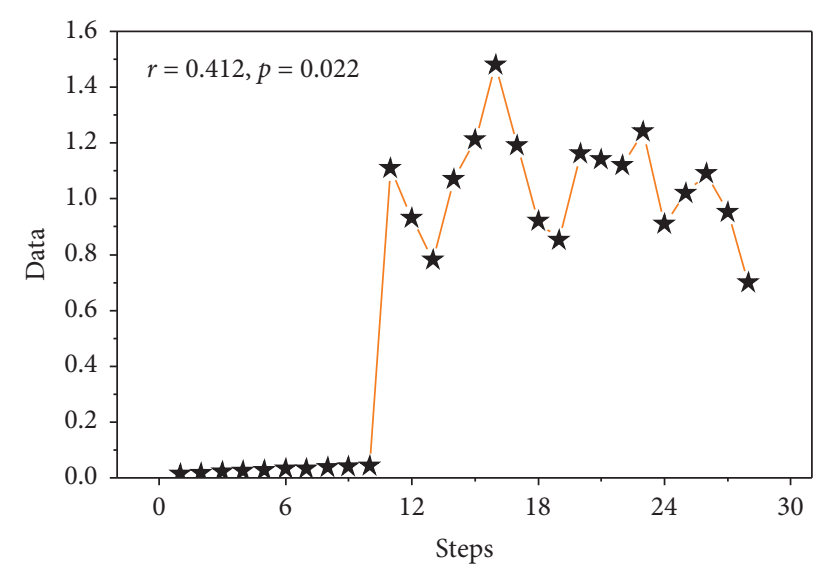

(d)

Figure 8: Changes in postoperative gait parameters.

early in the learning curve, a higher risk of complications, and a higher risk of intraoperative bleeding.

\section{Conclusion}

The DAA does not damage the muscles around the hip joint and does not cause any significant damage to the posterior structures of the hip capsule. The balance of muscle strength of the lower limbs after surgery is better than that of the traditional approach, so the accuracy of the placement of the prosthesis and the good stability of the hip joint after surgery reduce the risk of postoperative soft tissue imbalance caused by periprosthetic muscle injury and postoperative hip dislocation caused by improper placement of the prosthesis. Daily activities such as squatting, sitting on a low stool, and bending over to put on socks and laces are possible in the early postoperative period, and no special protection is needed for the postoperative hip joint. In this paper, we observed the angle of the posterior prosthesis using imaging and showed that total hip arthroplasty using the direct anterior approach resulted in better hip mobility and less pain in the early postoperative period compared with other approaches, but there was no advantage in incision length between total hip arthroplasty using the direct anterior approach and the direct lateral and posterior lateral approaches and some of the data (operative time, blood loss, etc.) were significantly worse than those using the direct anterior approach. In addition, the direct anterior approach to total hip arthroplasty is subject to a learning curve and requires at least 33 cases of experience to achieve a lower complication rate.

\section{Data Availability}

The data used to support the findings of this study are available from the corresponding author upon request.

\section{Conflicts of Interest}

The authors declare that they have no known competing financial interests or personal relationships that could have appeared to influence the work reported in this paper.

\section{Acknowledgments}

The study was supported by the Special Fund for Youth Clinical Research of Peking University First Hospital (2019CR27).

\section{References}

[1] A. Bengtsson, G. S. Donahue, S. Nemes, G. Garellick, and O. Rolfson, "Consistency in patient-reported outcomes after total hip replacement," Acta Orthopaedica, vol. 88, no. 5, pp. 484-489, 2017.

[2] H. S. Na, H. J. Shin, Y. J. Lee, J. H. Kim, K. H. Koo, and S. H. Do, "The effect of tranexamic acid on blood coagulation in total hip replacement arthroplasty: rotational thromboelastographic (ROTEM) analysis," Anaesthesia, vol. 71, no. 1, pp. 67-75, 2016.

[3] X. Wang, D. J. Hunter, G. Vesentini et al., "Technologyassisted rehabilitation following total knee or hip replacement for people with osteoarthritis: a systematic review and metaanalysis," Bmc Musculoskeletal Disorders, vol. 20, no. 1, pp. 506-516, 2019.

[4] J. Zhang, "Research progress of total hip arthroplasty through direct approach by lateral position," China Clinical Practical Medicine, vol. 8, no. 3, pp. 11-13, 2017.

[5] S. Lyman, Y.-Y. Lee, P. D. Franklin, W. Li, D. J. Mayman, and D. E. Padgett, "Validation of the HOOS, jr: a short-form hip replacement survey," Clinical Orthopaedics \& Related Research, vol. 474, no. 6, pp. 1472-1482, 2016.

[6] M. Figueroa-Labastida and A. Farooq, "Simultaneous lateral and endwall high-speed visualization of ignition in a circular shock tube," Combustion and Flame, vol. 214, pp. 263-265, 2020.

[7] N. M. Kitchen and R. C. Miall, "Proprioceptive deficits in inactive older adults are not reflected in fast targeted reaching movements," Experimental Brain Research, vol. 237, no. 2, pp. 531-545, 2019.

[8] T. Hoff, "The battle of the bundle: lessons from my mother's partial hip replacement," Health Affairs, vol. 36, no. 8, pp. 1511-1514, 2017.

[9] A. Lübbeke, A. J. Silman, C. Barea, D. Prieto-Alhambra, and A. J. Carr, "Mapping existing hip and knee replacement registries in Europe," Health Policy, vol. 122, no. 5, pp. $548-557,2018$. 
[10] W. P. R. Melman, B. P. Mollen, B. J. Kollen, and C. C. P. M. Verheyen, "First experiences with the direct anterior approach in lateral decubitus position: learning curve and 1 year complication rate," Hip International, vol. 25, no. 3 , pp. 251-257, 2015.

[11] F. Wirthmüller, J. Schlechtriemen, J. Hipp et al., "Teaching vehicles to anticipate: a systematic study on probabilistic behavior prediction using large data sets," IEEE Transactions on Intelligent Transportation Systems, pp. 11-16, 2020.

[12] C. Delgado, K. Dawson, B. Schwaegler, R. Zachariah, S. Einav, and L. Bollag, "Hand placement during chest compressions in parturients: a pilot study to identify the location of the left ventricle using transthoracic echocardiography," International Journal of Obstetric Anesthesia, vol. 43, pp. 31-35, 2020.

[13] C. Foissey, C. Batailler, C. Fary, F. Luceri, E. Servien, and S. Lustig, "Transitioning the total hip arthroplasty technique from posterior approach in lateral position to direct anterior approach in supine position-risk factors for acetabular malpositioning and the learning curve," International Orthopaedics, vol. 44, no. 9, pp. 1669-1676, 2020.

[14] H. Ike, L. D. Dorr, N. Trasolini, M. Stefl, B. McKnight, and N. Heckmann, "Spine-pelvis-hip relationship in the functioning of a total hip replacement," Journal of Bone and Joint Surgery, vol. 100, no. 18, pp. 1606-1615, 2018.

[15] R. Blomfeldt, H. Törnkvist, K. Eriksson, A. Söderqvist, S. Ponzer, and J. Tidermark, "A randomised controlled trial comparing bipolar hemiarthroplasty with total hip replacement for displaced intracapsular fractures of the femoral neck in elderly patients," The Journal of Bone and Joint Surgery. British Volume, vol. 89-B, no. 2, pp. 160-165, 2007.

[16] H. F. Xu, R. S. White, D. L. Sastow, M. H. Andreae, L. K. Gaber-Baylis, and Z. A. Turnbull, "Medicaid insurance as primary payer predicts increased mortality after total hip replacement in the state inpatient databases of California, Florida and New York," Journal of Clinical Anesthesia, vol. 43, pp. 24-32, 2017.

[17] A. Borjali, A. F. Chen, O. K. Muratoglu, M. A. Morid, and K. M. Varadarajan, "Detecting total hip replacement prosthesis design on plain radiographs using deep convolutional neural network," Journal of Orthopaedic Research, vol. 38, no. 7, pp. 1465-1471, 2020.

[18] B. I. Eriksson, L. Borris, O. E. Dahl et al., "Oral, direct Factor Xa inhibition with BAY 59-7939 for the prevention of venous thromboembolism after total hip replacement," Journal of Thrombosis and Haemostasis, vol. 4, no. 1, pp. 121-128, 2006.

[19] E. Lenguerrand, M. R. Whitehouse, A. D. Beswick et al., "Risk factors associated with revision for prosthetic joint infection after hip replacement: a prospective observational cohort study," The Lancet Infectious Diseases, vol. 18, no. 9, pp. 1004-1014, 2018.

[20] M. R. Lassen, A. Gallus, G. E. Raskob, G. Pineo, D. Chen, and L. M. Ramirez, "Apixaban versus enoxaparin for thromboprophylaxis after hip replacement," New England Journal of Medicine, vol. 363, no. 26, pp. 2487-2498, 2010.

[21] R. S. Camenzind, K. Stoffel, N. J. Lash, and M. Beck, "Direct anterior approach to the hip joint in the lateral decubitus position for joint replacement," Operative Orthopädie und Traumatologie, vol. 30, no. 4, pp. 276-285, 2018.

[22] E. Burn, C. J. Edwards, D. W. Murray et al., "The impact of $\mathrm{BMI}$ and smoking on risk of revision following knee and hip replacement surgery: evidence from routinely collected data," Osteoarthritis and Cartilage, vol. 27, no. 9, pp. 1294-1300, 2019.
[23] A. H. Shadyab, R. Terkeltaub, C. Kooperberg et al., "Prospective associations of C-reactive protein (CRP) levels and CRP genetic risk scores with risk of total knee and hip replacement for osteoarthritis in a diverse cohort," Osteoarthritis and Cartilage, vol. 26, no. 8, pp. 1038-1044, 2018.

[24] A. Emmert, R. Franke, I. F. Brandes et al., "Comparison of conductive and convective warming in patients undergoing video-assisted thoracic surgery: a prospective randomized clinical trial," Thoracic and Cardiovascular Surgeon, vol. 65, no. 5, pp. 362-366, 2017.

[25] T. E. Lindheimer, K. Fitzpatrick, R. Avelar et al., "Effect of geometric factors on lateral position of vehicles in freeway buffer-separated managed lanes," Transportation Research Record, vol. 2616, no. 2616, pp. 10-18, 2017. 